\title{
3D-reconstruction of imperfect noisy helical particles: The structure of individual frozen-hydrated HbS fibers.
}

\author{
G.Kishchenko and R.Josephs \\ Molecular Genetics and Cell Biology. University of Chicago, Chicago, IL 60637
}

Deoxygenated sickle cell hemoglobin polymerizes in red cells to form helical fibers approximately $250 \AA$ in diameter and several microns long. These fibers fill the red cell and make it rigid. Cells filled with fibers block capillaries reducing blood flow thereby causing the symptoms of sickle cell disease. We have been studying the molecular structure of these fibers by cryoelectron microscopy and image reconstruction in order to determine the intermolecular contacts that stabilize the fibers.

Sickle hemoglobin fibers have several characteristics that make their analysis uniquely challenging. The Fourier-Bessel transform algorithm for 3D-reconstruction of helical particles assumes that particles are straight and have constant pitch. Sickle hemoglobin $(\mathrm{HbS})$ fibers deviate from this ideal in that they are curved and have a variable pitch. The resolution of reconstructions can be improved by averaging multiple particles. For averaging one must determine the relative orientation and scaling factor since the magnification of the microscope will vary over time. Because $\mathrm{HbS}$ fibers are not straight and have a variable pitch the determination of their orientation is rendered more difficult than in the case of better behaved particles. The low signal/noise ratio characteristic of micrographs of frozen hydrated specimens makes it difficult to locate the particle axis and its boundaries.

We developed following procedures to deal with these issues. (1) Because the projection of the fiber onto a line perpendicular to the fiber axis is centrosymetric the imaginary part of the 1-dimensional Fourier transform (FT) of the fiber projection is zero if the phase origin is located on the particle axis. Thus a systematic shift of the phase origin to minimize the imaginary part of the FT of the projection provides a very good estimate of the axis location. (2) To obtain high precision scaling we used the position of a meridional $31.65 \AA$ peak in the FT. This is the strongest peak in the transform and corresponds to the subunit rise. In order to assure that an exact repeat distance was being used we varied the length of the 'box' bounding the particle and selected that length which produced the highest amplitude for this reflection. (3) If a particle is not straight then its 1-dimensional projection is not centrosymetric. This leads to a non zero value for the imaginary part of the 1-dimensional FT. Thus the straightest particles were selected by minimizing the value of the amplitude of the imaginary part of the 1-dimensional FT of the axial 1D projection referred to above. (4) Within a length of one repeat, approximately $3000 \AA$, the variation in pitch was small enough to not cause problems in the reconstruction. The pitch was estimated from the subunit rise and repeat length. As an additional check we also used different values for the pitch as a parameter for the reconstruction procedure. The best estimate of the pitch gave the highest contrast of reconstructed helical projection and also corresponde to the value determined from the repeat length and subunit rise.

The above approach provided a reconstruction with a resolution of $32 \AA$. However when six particles were averaged the resolution improved to $16 \AA$ radially and $8 \AA$ axially.

This work was supported by NIH grant HL58512 


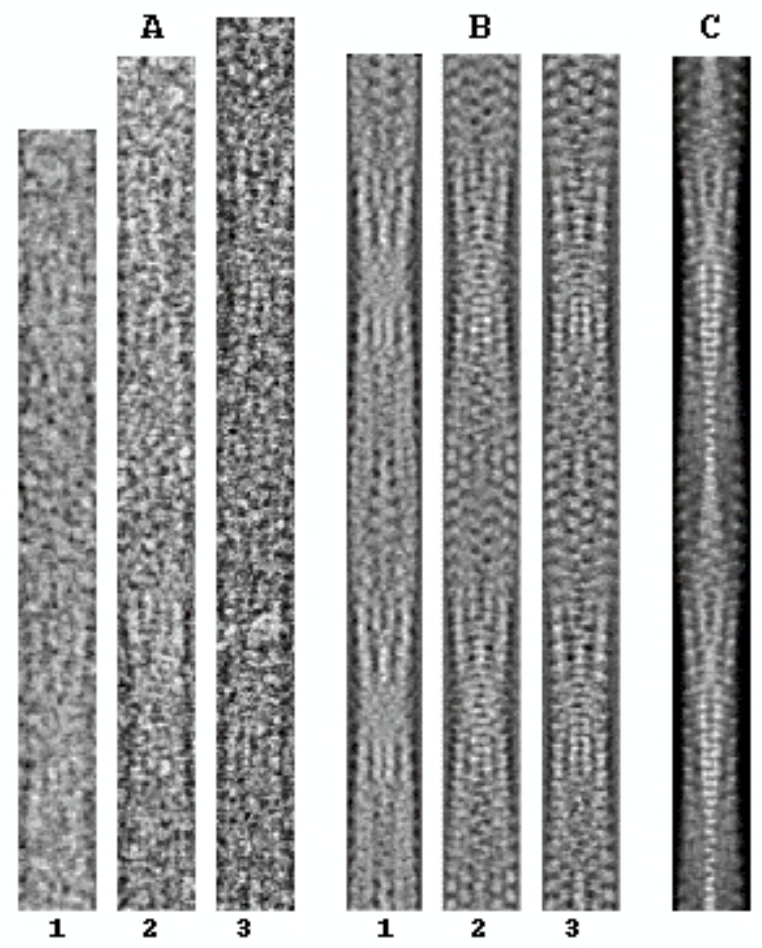

Fig. 1

a. Micrographs of frozen-hydrated sickle hemoglobin fibers;

b - lateral projections of 3D-reconstructions of the same fibers;

c - the average lateral projection obtained by averaging of 6 reconstructions.

side view

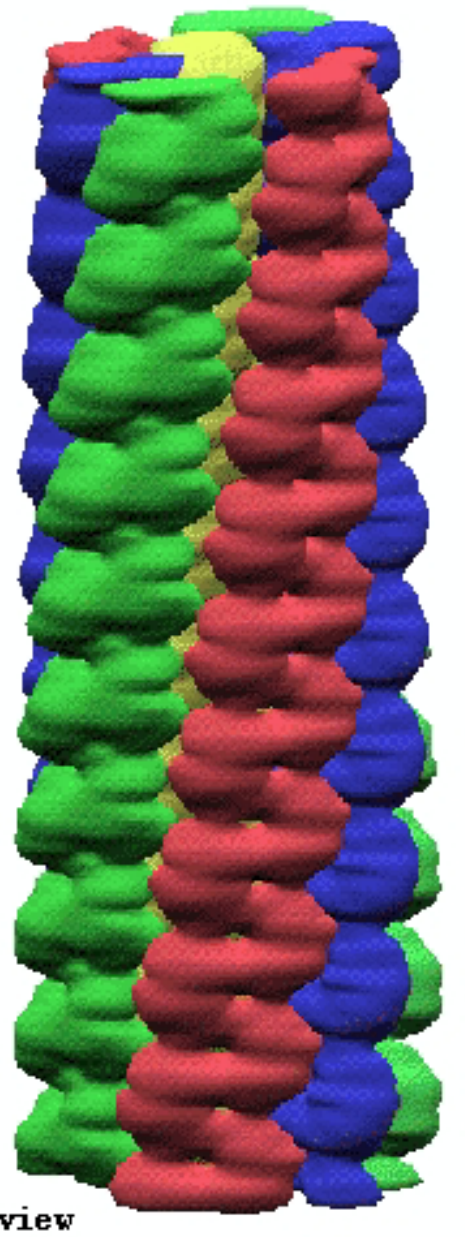

view from the top

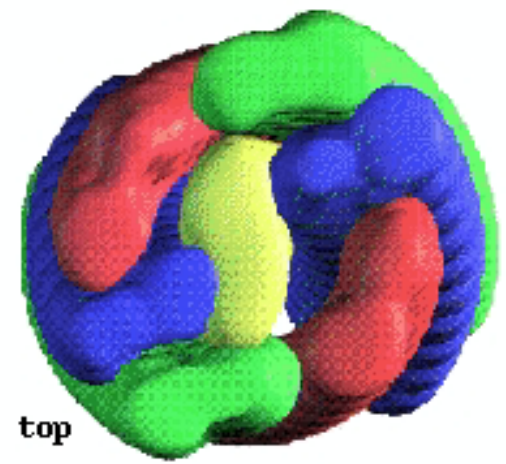

Fig. 2

Computer rendering of average $3 \mathrm{D}$-reconstruction of $\mathrm{HbS}$ fiber.

The hemoglobin molecule form pairs of strands (called double strands) in which the Hb molecules are stagcered by one half of the subunit rise, $31.65 \AA$. $A$ fiber consists of seven double strands twisted about a common helical axis. colors indicate the different double strands. 